\title{
Scheduling Data Transmissions of Underwater Sensor Nodes for Maximizing Value of Information
}

\author{
Ladislau Bölöni, Damla Turgut \\ Dept. of Electrical Engineering and Computer Science \\ University of Central Florida \\ Orlando, FL 32816-2450 \\ \{turgut,lboloni\}@eecs.ucf.edu
}

\author{
Stefano Basagni \\ ECE Department \\ Northeastern University \\ Boston, MA 02115 \\ basagni@ece.neu.edu
}

\author{
Chiara Petrioli \\ Dipartimento di Informatica \\ Università di Roma "La Sapienza" \\ Rome, Italy \\ petrioli@di.uniroma1.it
}

\begin{abstract}
We consider an underwater wireless sensor network where baseline communication happens over acoustic, multihop routes from the underwater nodes to an on-shore station. The data collected by the nodes greatly exceeds the baseline communication capability. At best, the nodes can transmit digests of their full observations. In order for the sink to receive all sensed data, an autonomous underwater vehicle (AUV) is sent to each node for collecting data over short-distance, high data rate optical connections. The AUV then offloads all collected information to the terrestrial station via wireless communication when it surfaces. The observations made by the nodes vary in size and urgency. The information they provide has an associated value. Given a path of the AUV, we design scheduling strategies for the nodes to decide when and how much information (i.e., which digest) to transmit via acoustic routes so that the value of information reaching the terrestrial station is maximized. These strategies are compared via simulations on realistic scenarios. Our results show that scheduling algorithms that are able to locally estimate the value of information of a data digest provide the delivery of data with a significantly higher value of information. In contrast, uninformed algorithms, i.e., strategies that do not consider the value of information at the node level, provide only a marginal increase over the benchmark case of using only the AUV for data collection.
\end{abstract}

\section{INTRODUCTION}

As the waters cover well over $70 \%$ of the earth surface, exploring the underwater world has always been at the forefront of human interests. While accessing the seas and their depths has been always a challenge, recent advances in communications and sensing technologies have favored remarkable improvements on the gathering of critical information about what lies below the surface. For instance, monitoring stations, deployed and then retrieved periodically for data collection through expensive expeditions at sea, were progressively substituted by wired interconnection of the submerged stations, allowing for real-time information gathering. Since underwater cabling is extremely expensive, research is now investigating the use of underwater wireless sensor networks (UWSNs) for data sensing and communications [1].

This paper concerns the problem of wireless data collection in a UWSN where a number of sensor nodes (SNs) are deployed on the ocean floor throughout an area of several square $\mathrm{km}$. The SNs use a variety of sensors, the highest data rate being provided by high definition video cameras (with data rates in the order of several Mbps). SNs have dual

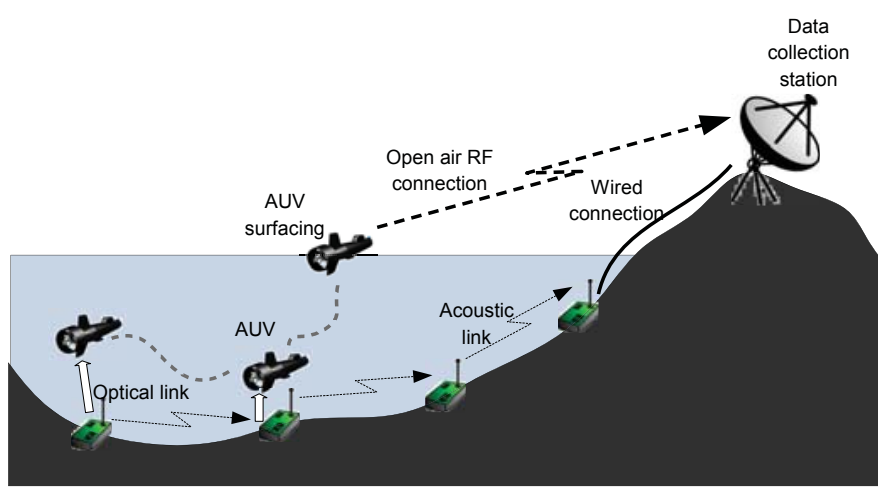

Fig. 1: An UWSN where nodes have dual communication capabilities.

communication capabilities [2]. The primary communication mode of the SNs is using an acoustic modem to send data to a data collection station located on shore. Communication is multi-hop to an SN that is directly connected to the station. This mode involves relatively low latency in delivering data, since as soon as a packet is crafted, it can be transmitted. However, this form of communication is prone to high BER and limited to a communication bandwidth currently below $10 \mathrm{kbps}$. For this reason, an SN can transmit at most a digest of the collected data over the acoustic links. The secondary communication method involves the use of an autonomous underwater vehicle (AUV) [3]. The AUV visits the SNs and downloads their data using high speed/short distance optical communication [4]. Eventually, the AUV offloads the collected data by periodically surfacing and transmitting them to the terrestrial station using over-the-air wireless communications. Fig. 1 illustrates the considered scenario.

Experience shows that the size, value and urgency of the information collected by the SNs can widely vary. It is also unpredictable to establish when and where a valuable event will happen and which node will be able to sense and report it. In any case, the original value of the sensed information decreases with time, and as such it should be delivered to the terrestrial station, and eventually to the customer, as soon as possible: The later the data reaches the customer, the lower its value will be. Therefore, each SN must decide when a less 
valuable digest (e.g., a lower resolution link or a photo) of a sensed data chunk should be sent to the terrestrial station via an acoustic route, and which digest among many of that chunk should be transmitted first.

In this paper we develop models for the value of information (VoI) of data chunks resulting from underwater observation, investigate the complexity of designing scheduling strategies for acoustic transmissions, and we design different scheduling heuristics for the SNs to decide which data chunk to transmit and when, over the acoustic routes. The overall goal is to design scheduling strategies that maximize the VoI of the data delivered to the terrestrial station. Simulation studies on realistic scenarios show that scheduling algorithms that are able to locally estimate the VoI of a data chunk provide a significantly higher VoI of the delivered data. In contrast, uninformed algorithms, i.e., techniques that do not consider VoI at the node level, provide only a marginal increase over using only the AUV for data collection.

To the best of our knowledge, there is no previous research on scheduling strategies for maximizing the value of information from underwater SNs equipped with acoustic and optical modems. The scenario considered in this paper is similar to that described by Farr et al. in [2], [5]. In particular, [2] describes the system of integrated optical and acoustic capabilities. In [5] an application is shown where an AUV is sent to offload data from a seafloor borehole observatory. The focus of these works is that of demonstrating the feasibility and effectiveness of deploying optical and acoustic technologies jointly for efficient underwater communication, enabling application (e.g., those involving images and videos) that would not be well supported by acoustic communications alone. Our work has been inspired by the scenarios described in these papers.

The remainder of this paper is organized as follows. Section II develops a formal model for the value profiles of information chunks collected by the nodes. A value profile describes the value of information of the data, its digests of various sizes, and its decay in time. In Section III, we devise transmission scheduling algorithms for the SNs. Section IV reports the results of simulation-based experiments providing a comparative performance evaluation of the proposed algorithms. We present concluding remarks in Section V.

\section{VALUE OF INFORMATION IN UWSNS}

\section{A. A formalism for the value of information}

The metric of value of information has been originally proposed in game theory as the price an optimal player would pay for a piece of information [6]. In the context of sensor networks, a number of recent projects have introduced similar metrics to model situations where one either needs to select a subset of the collected data or choose between transmitting a piece of information or not [7], [8], [9].

The formalism of the value of information collected in an UWSN we present will also allow the consideration of the decay of information in time and the value of digests of information chunks. We assume that the data recorded at a given $\mathrm{SN}$ is collected in a data chunk $D$ of size $|D|$. We denote with $t_{r}(D)$ the time at which the data chunk was created. Naturally, the information in the data chunk refers to observations before this time. We denote with $\mathbb{D}$ the set of all possible data chunks.

A digest $\operatorname{dig}(D, b)$ of size $b$ is a subset of information of data chunk $D$. When $b>|D|$ the digest is assumed to be the original data chunk itself.

With $\mathcal{V}(D, t)$ we indicate the value of information (VoI) of the data chunk $D$ received by the terrestrial station (customer) at time $t$. We will give a pragmatic definition of this value, by relating it to the value $\mathcal{V}(a)$ of the action $a$ taken by the customer.

Definition 1: The value of information $\mathcal{V}(D, t)$ is the sum of the values of all actions $a_{i}$ of the customer taken according to a customer strategy $s$ for which the knowledge of $D$ is an informational prerequisite:

$$
\mathcal{V}(D, t)=\max _{s} \sum_{a_{i}} \mathcal{V}\left(a_{i}\right) .
$$

By giving a definition of VoI in pragmatic terms, as the value of the actions taken by the customer as a consequence of receiving information, we need to consider whether the actions could have been taken without this piece of information. For instance, it is possible that the customer received the same information from another $\mathrm{SN}$, or that it previously received a digest of the same data chunk.

Definition 2: The conditional value of information $\mathcal{V}\left(D, t \mid\left(D_{1}, t_{1}\right) \ldots\left(D_{k}, t_{k}\right)\right)$ is the value of receiving data chunk $D$ at time $t$ given that data chunks $D_{i}$ had been previously received at time $t_{i}$ respectively.

The value is defined pragmatically as the difference between the value for a customer who did not receive the previous values, and one who did. We can immediately infer a property:

Property 1: The sum of the VoI of any combination of digests is smaller than sending the original data chunk $D$ at the time of transmission of the first data digest. Let us consider a series of strictly increasing points in time $t_{1} \ldots t_{n}$ and corresponding strictly increasing digest levels $b_{1} \ldots b_{n}$. Then:

$$
\begin{aligned}
& \sum_{i=0}^{n} \mathcal{V}\left(\operatorname{dig}\left(D, b_{i}\right), t_{i} \mid\left(\operatorname{dig}\left(D, b_{1}\right), t_{1}\right) \ldots\left(\operatorname{dig}\left(D, b_{i-1}\right), t_{i-1}\right)\right) \\
& \quad \leq \mathcal{V}\left(\operatorname{dig}\left(D, b_{n}\right), t_{1}\right)
\end{aligned}
$$

Proof: Let us consider two consumers $C_{1}$ and $C_{2}$ with optimal strategies. $C_{1}$ receives the increasing digests at the given points in time $t_{1} \ldots t_{n}$, while $C_{2}$ receives the largest digest $b_{n}$ at the initial time point $t_{1}$. By contradiction, let us assume that the customer $C_{1}$ receives a $\mathrm{VoI}$ strictly higher than $C_{2}$. However, since every smaller digest can be extracted from larger digests, $C_{2}$ can choose the following strategy: At any point $t_{i}$ extract the corresponding digest $b_{i}$ and then use the same strategy as $C_{1}$. In this way $C_{2}$ performs the same actions, and thus obtains the same VoI of $C_{1}$, a contradiction. 


\section{B. Forms of value of information}

We are interested in finding a closed form expression for the $\mathrm{VoI}$ of a digest $\operatorname{dig}(D, b)$ at time $t$. This value is going to be lower than the initial value $\mathcal{V}\left(D, t_{r}\right)$ because of the urgency of information, which makes the VoI decrease in time, and the summarizability, which describes how the VoI decreases when the data is replaced with a digest. In the remainder of this paper we will always assume that urgency and summarizability are independent from each other. This is justified by the observation that the urgency depends on the meaning of the observation (the witnessed real-world event) while the summarizability of the data chunk largely depends on the structure of the data. This assumption allows us to write the $\mathrm{VoI}$ in the following form:

$$
\mathcal{V}(\operatorname{dig}(D, b), t)=d_{d}\left(\frac{b}{|D|}\right) \cdot d_{t}\left(t-t_{r}\right) \cdot \mathcal{V}(D, t),
$$

where $d_{d}$ is the digest value decay function and $d_{t}$ is the temporal value decay function. Without loss of generality, we define $d_{d}$ as the ratio between digest size and original data chunk size, while we define $t_{r}$ to be the time of the data collection.

It would be convenient to make the assumption that $d_{d}$ and $d_{t}$ have unique system-wide definitions. Unfortunately, realworld experience does not confirm this: The urgency varies with the observed events and the summarizability varies with the sensor types and the specific sensing parameters used by the SN for a given data chunk. We will, however, make a weaker assumption: We will assume that we know the parameterized form of these functions. We will call the VoI profile of a data chunk the triplet of the initial value, the digest value decay function and the temporal value decay function.

We model the temporal value decay function using an exponential decay model:

$$
d_{t}\left(t-t_{0}\right)=\exp \left(P_{t d} \cdot\left(t-t_{0}\right)\right)
$$

where $P_{t d}$ is the temporal decay parameter. In particular, $P_{t d}=0$ indicates that the information is not delay sensitive, and $P_{t d} \gg 0$ represents a very fast decay of information. This model can fit many practical applications of UWSNs.

Let us now consider the form of the digest value decay function $d_{d}$. Although the size parameter $b$ might imply a continuous function, in practice the creation of digests might imply a combination of different approaches. For instance, the creation of a digest from a video stream can progress through reduction of resolution, reduction of frame-rate, extracting key frames, various techniques for lossy encoding and output of image recognition and object tracking techniques. Thus, we can characterize the value in form of a piecewise constant function, which can be described with a series of number pairs $\left\{\left(d_{i}, v_{i}\right)\right\}$.

\section{Conditional VoI}

Intuitively, the conditional VoI represents the novel information provided by the new digests assuming that smaller digests have been received previously. If all digests would have been

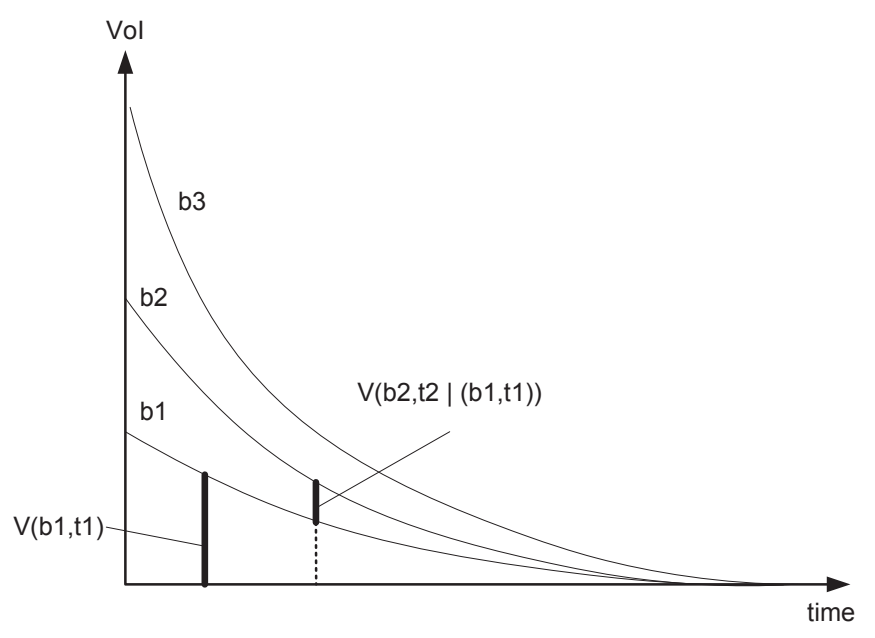

Fig. 2: The conditional VoI is the difference between the unconditional $\mathrm{VoI}$ and the VoI of previously transmitted digests at the current time.

received at exactly the same moment, the result would be the difference between the two realized values. However, as the smaller digests have been received earlier, we will not subtract their arrival-time values, but the value they would have at the current moment. Fig. 2 illustrates this with the example of a digest of size $b_{1}$ received at time $t_{1}$ and a larger digest of size $b_{2}$ arriving at a later time $t_{2}$. The conditional VoI is expressed by the following formula:

$$
\begin{aligned}
& \mathcal{V}\left(\operatorname{dig}\left(D, b_{2}\right), t_{2} \mid\left(\operatorname{dig}\left(D, b_{1}\right), t_{1}\right)\right)= \\
& \quad \mathcal{V}\left(\operatorname{dig}\left(D, b_{2}\right), t_{2}\right)-\mathcal{V}\left(\operatorname{dig}\left(D, b_{1}\right), t_{2}\right) .
\end{aligned}
$$

\section{TRANSMISSION SCHEDULING ALGORITHMS}

All SNs continually make observations and record them at specific points in time $\left(D_{1}, t_{1}\right), \ldots,\left(D_{i}, t_{i}\right), \ldots$ We assume that an SN has no memory limitation and can store all the observation it makes till the AUV comes. SNs use the acoustic modem to transmit digests of the data chunk they collect. When the AUV visits an SN, all the data chunks the node has collected since the previous visit of the AUV are transferred to the AUV (optically). Whenever a transmission has finished, the node must schedule a new digest for transmission. Deciding which digest to transmit and when determines the VoI received at the terrestrial station. The overall aim of designing a scheduling algorithm is that of maximizing the total VoI of the data delivered. Note that the transmission scheduling on the acoustic link also affects the VoI of the data carried by the AUV, which carries data whose value is the conditional VoI of the previously transmitted digests.

In this section we start by investigating the computational complexity of scheduling digest transmissions over the acoustic link, and then we define heuristics for solving the problem. 


\section{A. The complexity of scheduling}

We start by investigating the complexity of a simplified version of the scheduling problem. Let us assume that we have a static collection of data chunks, times and profiles $\left\{\left(D_{1}, t_{1}, P_{1}\right) \ldots\left(D_{n}, t_{n}, P_{n}\right)\right\}$, and a time interval $\left[t_{s}, t_{d}\right]$. No new chunks will be recorded after $t_{d}$ and none of the chunks or any of the digests have been transmitted prior to $t_{s}$. We assume that the node transmits over a uniform bandwidth $B$.

The scheduling problem we are trying to solve is to find a schedule, i.e., an ordered list of tuples $S=\left\{\left(j_{i}, b_{i}\right)\right\}$, such that the $i$-th transmission on the acoustic link will be the sending of $\operatorname{dig}\left(D_{j_{i}}, b_{i}\right)$ at the time:

$$
t_{i}=\frac{\sum_{j=1}^{i-1} b_{j}}{B} .
$$

We want to maximize the total value of information that is transmitted:

$$
\mathcal{V}(S)=\sum_{i} \mathcal{V}\left(\operatorname{dig}\left(D_{j_{i}}, b_{i} \mid \Xi_{i}\right),\right.
$$

where $\Xi_{i}$ are the previous transmissions of digests of $D_{j_{i}}$ in schedule $S$.

Property 2: The scheduling problem is NP hard.

Proof: Let us assume that the problem is not NP hard. The optimization version of the knapsack problem, which is known to be NP hard, can be seen as a case of the scheduling problem where all the digests are worth zero, and the decay parameter of the function is zero (no decay in time). Thus, an algorithm which would solve the scheduling problem in polynomial time would also solve the knapsack problem, which is a contradiction.

Since this simplified version of the problem is computationally hard, so is the problem of scheduling the transmission of digests from a continuous generation of data chunks. In the following, we define three practically deployable transmission scheduling heuristics.

\section{B. Heuristic 1: AUV only (AUVO)}

A first solution to our problem of scheduling digests for acoustic transmissions is that of not scheduling them at all: Data are delivered to the terrestrial station only by successive surfacing of the AUV. This heuristic, termed AUV only, or AUVO, will be used as a benchmark solution against the following ones, where the use of acoustic modems and multihop underwater routes is allowed. In AUVO, the VoI received by the customer is the VoI of the data chunks collected by the AUV at the moment when they were delivered. We notice that any strategy that through the use of acoustic routes will succeed in delivering pieces of data chunks before the AUV delivers them will improve the VoI of the collected data. Nevertheless, AUVO has some significant practical advantages. For instance, the SNs will be considerably cheaper, as they will not need to be equipped with expensive acoustic modems (in the tens of thousands of dollars each); the SNs will have longer battery life, as acoustic transmissions consume

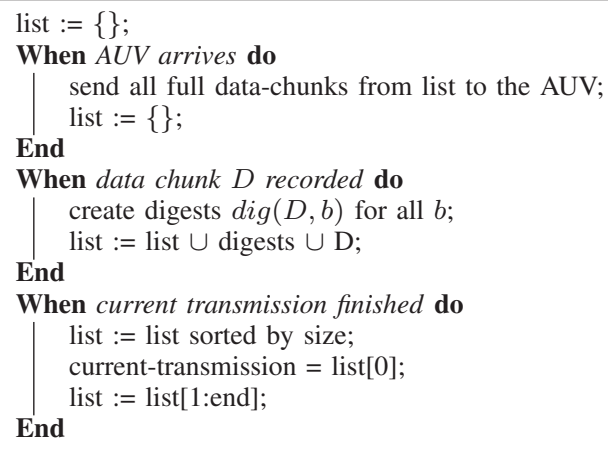

Fig. 3: Uninformed Progressive Digest scheduling

significant energy resources; finally, the protocol stack of the node will be considerably simpler, since no underwater MAC and routing function are needed when communication reduce to offloading data on the one-hop optical link.

\section{Heuristic 2: Uninformed Progressive Digest (UPD)}

In the Uninformed Progressive Digest (UPD) strategy an SN cannot perform a local evaluation of the VoI profile of the data chunks (hence its uninformed character). However, it knows the shape of the $d_{t}$ function and also knows that the steps of the digests are arranged in such a way that the $\mathrm{VoI}$ decreases slower than the size of the digest. This being all the knowledge a node has, its best choice is to send the smallest available digests at any given moment, followed by a digest a step larger, and so on, breaking the ties via the arrival time. The resulting scheduling algorithm is described in Fig. 3.

\section{Heuristic 3: Greedy Average VoI (GAVI)}

The Greedy Average VoI (GAVI) strategy assumes that an $\mathrm{SN}$ is able to identify the value profile of the sensed data chunks. With this information, the strategy greedily maximized the average VoI transmitted. Scheduling the transmissions one-by-one, the next transmission will be the one for which the VoI per unit of time is the highest. The VoI considered here is the conditional VoI taking into consideration previous transmissions of the same data chunk. For a static set of data chunks, GAVI is actually an optimal strategy. However, for a node which constantly receives new data chunks, it can happen that a new, higher value digest might have to wait because a longer data chunk is currently in transmission. The algorithm is described in Fig. 4.

\section{Simulation RESUlts}

We demonstrate the effectiveness of our algorithms for maximizing the VoI of collected data through simulations. We consider a network with $40 \mathrm{SNs}$ deployed in a grid over a $6 \times 7 \mathrm{~km}^{2}$ area of ocean floor at an average distance of $1500 \mathrm{~m}$ from each other. Each node is outfitted with an acoustic modem. We make the assumption that the average transfer rate between a node and the sink is 10kbps. The AUV has been modeled after an Odyssey-IV class vehicle, with a cruise speed $1.8 \mathrm{~m} / \mathrm{s}$ [3]. As a result, the AUV takes around 13 minutes to travel between two SNs. Optical communication can reach a 


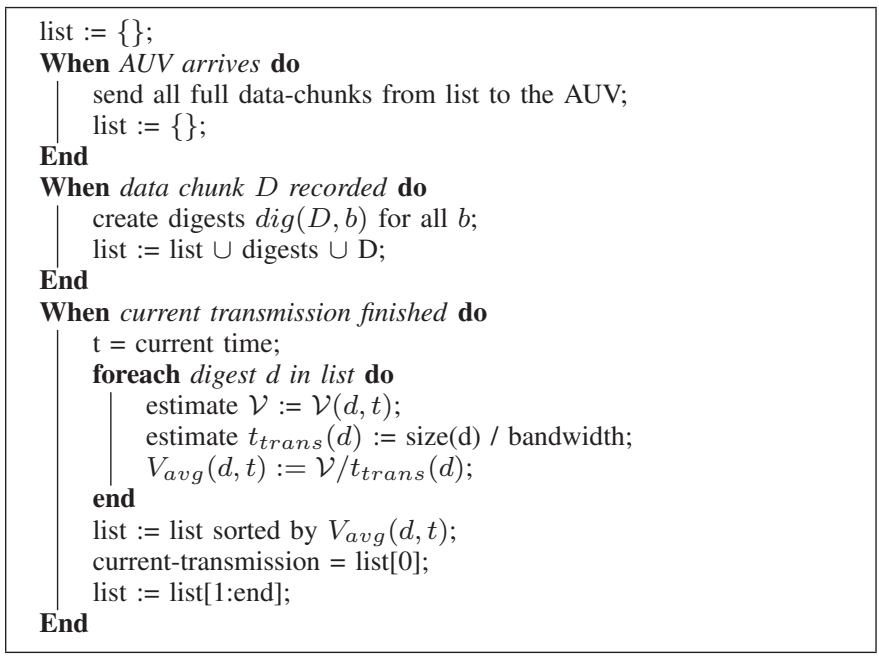

Fig. 4: Greedy Average VoI scheduling

TABLE II: Observation classes, base $\operatorname{VoI} \mathcal{V}(D, t)$ and temporal decay $P_{t d}$.

\begin{tabular}{|l|l|l|l|}
\hline Type & Description & $\mathcal{V}(D, t)$ & $P_{t d}$ \\
\hline C1 & $\begin{array}{l}\text { No specific event } \\
\text { C2 }\end{array}$ & $\begin{array}{l}\text { Low priority event (for instance, movement } \\
\text { of a shoal of fish or sea mammals) }\end{array}$ & 1.0 \\
C3 & $\begin{array}{l}\text { High priority event (the camera captured } \\
\text { divers, AUVs or submarines) }\end{array}$ & 10.0 & 1.0 \\
\hline
\end{tabular}

data rate of $10 \mathrm{Mbps}$ when the AUV hovers less than $100 \mathrm{~m}$ from the SN (clear water communication) [2]. In order for the AUV to download the $1.1 \mathrm{~GB}$ collected by an SN in a day, it has to hover or circle around an SN [10] for about 13 minutes. In this scenario the AUV visits all $40 \mathrm{SNs}$ in fixed order over the course of a day, then it returns to its dock for refueling and offloading the data.

We simulate a scenario where the SNs use cameras to take videos of the deployment area for intrusion detection purposes. The SNs store surveillance data in the form of $720 \mathrm{p}$ high definition video (for a resolution of $1280 \times 720$ pixels, at $29.97 \mathrm{frame} / \mathrm{s})$. The recordings are partitioned into data chunks corresponding to 1 minute of recorded video. We assume that the video is encoded using the standard H.264 codec. We consider 4 types of digests, each representing one or several images extracted from keyframes in the video, encoded using the WEBP codec. We have assigned the $d_{d}$ digest values for these data based on a subjective evaluation of how much information can be extracted from the various images and how useful this information can be for customer. These values, and the value of other parameters, are listed and described in Table I.

In order to determine the value profiles of the data chunks, we divide them into three observation classes $\mathrm{C} 1, \mathrm{C} 2$ and $\mathrm{C} 3$, each of them having different urgency and basis information levels, as shown in Table II. The distribution of observation classes is not uniformly random: For instance, intruders have a tendency to linger longer than 1 minute in the area covered by a $\mathrm{SN}$. To account for this, we modeled the distribution

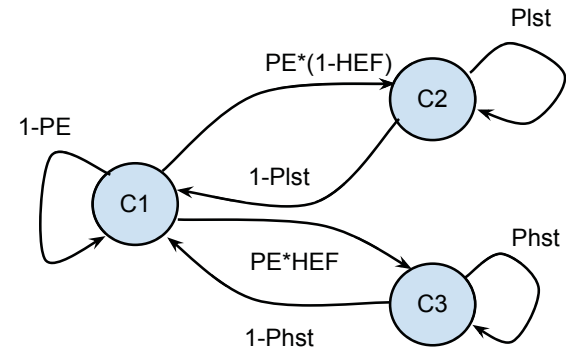

Fig. 5: The Markov chain modeling the occurrence of events.

of observations using the Markov chain shown in Fig. 5. We assume that for the majority of the time, the system will make observations of unimportant events (C1). Events happen with a certain probability PE. A fraction HEF of these events are of high priority. The self transitions Plst and Phst determine the average length of the events.

In our experiments we varied the fraction HEF of events with the highest VoI and run the three heuristics AUVO, UPD and GAVI. For each setting, we have generated 10 scenarios using different random seeds and averaged the values. Results are shown in Figure 6.

Fig. 6a shows the total VoI received at the terrestrial station for different values of HEF. As expected, the VoI increases with the HEF for all the heuristics because of the high priority events have higher base information values. The AUVO strategy, which does not perform acoustic transmissions, provides the lowest VoI, while GAVI, using local knowledge of the VoI of individual digests, has the highest overall VoI.

We observe that AUVO and UPD show surprisingly close behavior, especially for lower values of HEF $(<0.15)$. If the local evaluation of VoI is not possible, that is, the node is unable to determine by itself whether it sensed something urgent and important, it appears more advantageous to rely only on the AUV for data collection, without using acoustic routes. We notice that while not noticeably lowering the VoI, AUVO would significantly lower system costs (e.g., of acoustic modems) and extend the lifetime of the network. On the other hand, if local estimation of the $\mathrm{VoI}$ is possible, the use of acoustic communication allows GAVI to double the VoI of data delivered to the station.

We have also investigated the composition of the VoI depending on how data reach the terrestrial station. Fig. $6 \mathrm{~b}$ and Fig. $6 \mathrm{c}$ show the composition of the VoI achieved by UPD and GAVI, respectively. The Total VoI curve is the same as the corresponding one in Fig. 6a. This value is the sum of the VoI of data sent through acoustic routes plus the conditional VoI obtained via the AUV. Notice that the (non-conditional) VoI for data delivered by the AUV is the same in both cases (this is the value of AUVO in Fig. 6a). Looking at the graph for UPD, we can see that the reason why the UPD and AUVO strategies are so close in performance for low HEF ratios is not necessarily because the value carried over the acoustic links is low, but because the drop from the non-conditional to the conditional value carried by the AUV almost completely 
TABLE I: Digest levels, digest creation methodology and the digest value decay functions.

\begin{tabular}{|l|l|l|l|l|}
\hline Type & Size & $b$ & $d_{d}$ & Description \\
\hline 720p H.264 @ 29.97fps & $7500 \mathrm{kB}$ & 1 & 1 & High-definition video, full information content \\
4 keyframes (WEBP q=0.85) & $60 \mathrm{kB}$ & 0.008 & 0.9 & Four keyframes, allow identification of beginning/ending of presence and movement direction \\
2 keyframes (WEBP q=0.85) & $30 \mathrm{kB}$ & 0.004 & 0.7 & Two keyframes, allow identification of movement direction \\
1 keyframes (WEBP q=0.85) & $15 \mathrm{kB}$ & 0.002 & 0.5 & Good quality still picture \\
1 keyframes (WEBP q=0.75) & $7.5 \mathrm{kB}$ & 0.001 & 0.4 & Low quality still picture, quality might impede identification \\
\hline
\end{tabular}

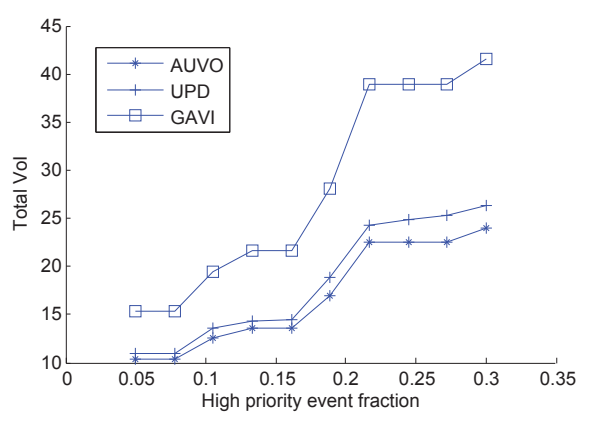

(a) Total value of information.

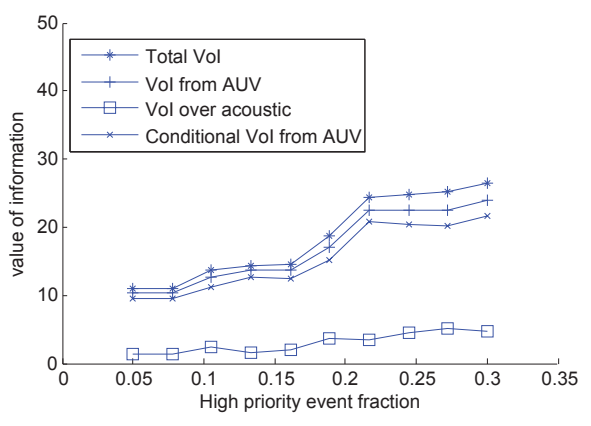

(b) UPD: VoI Distribution.

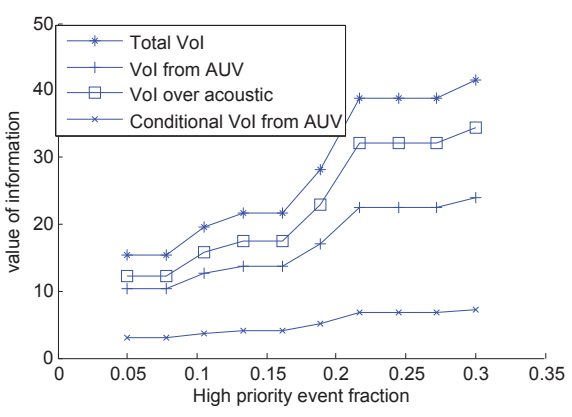

(c) GAVI: VoI Distribution.

Fig. 6: Value of Information: Total and composition function of HEF.

eliminates this gain. This is especially the case when most of the carried data is not urgent. This gain is instead maintained by GAVI, where a smarter, VoI-based choice of what to send acoustically is performed that delivers high VoI data earlier through acoustic routes.

\section{Conclusions And Future Work}

This paper concerns the definition and investigation of algorithms for scheduling the transmissions of information with a given, application-dependent value in UWSNs. In particular, in scenarios where nodes can transmit data over acoustic multihop routes and also via offloading them to a passing AUV, we have shown that it is possible to find scheduling strategies allowing the sensor nodes to acoustically transmit digests of the sensed data so that the value of information delivered to the customer is maximized. After proving the problem of acoustic scheduling computationally hard, we have defined two heuristics for acoustic data scheduling. These heuristics differ on the knowledge of the value of information to be transmitted. Through simulation results, we have shown that scheduling strategies that are able to locally compute the value of information of a data digest, like our GAVI, provide the delivery of data with a significantly higher value of information. Our uninformed algorithm, UPD, instead, provides only a marginal increase of VoI over the baseline case of using only the AUV for data collection.

In this paper we assumed that the AUV follows a fixed route to the SNs that is independent of the information collected at the nodes, and on its value. In fact, the AUV route has a major impact on the VoI of data delivered to the customer. Future work on this topic will consider route planning for one and multiple AUVs for maximizing VoI, as well as a comparative performance evaluation of different solutions.
Acknowledgments: This work was partially supported by the EC FP7 project CLAM (CoLlAborative eMbedded networks for submarine surveillance) for C. Petrioli, and two visiting faculty fellowships provided by University of Rome La Sapienza for D. Turgut and L. Bölöni.

\section{REFERENCES}

[1] T. Melodia, H. Khulandjian, L.-C. Kuo, and E. Demirors, "Advances in underwater acoustic networking," in Mobile Ad Hoc Networking: Cutting Edge Directions, S. Basagni, M. Conti, S. Giordano, and I. Stojmenovic, Eds. Hoboken, NJ: John Wiley \& Sons, Inc., March 5 2013, ch. 23, pp. 804-852.

[2] N. Farr, A. Bowen, J. Ware, C. Pontbriand, and M. Tivey, "An integrated, underwater optical/acoustic communications system," in Proceedings of the MTS/IEEE OCEANS 2010, Sydney, Australia, May 24-27 2010, pp. $1-6$.

[3] J. Eskesen, D. Owens, M. Soroka, and J. Morash, "Design and performance of ODYSSEY IV: a deep ocean hover-capable AUV," MIT Sea Grant, Tech. Rep. MITSG-09-08, 2009.

[4] F. Hanson and S. Radic, "High bandwidth underwater optical communication," Applied Optics, vol. 47, no. 2, pp. 277-283, 2008.

[5] N. Farr, J. Ware, C. Pontbriand, T. Hammar, and M. Tivey, "Optical communication system expands CORK seafloor observatory's bandwidth," in Proceedings of the MTS/IEEE OCEANS 2010, Seattle, WA, September 20-23 2010, pp. 1-6.

[6] R. A. Howard, "Information value theory," Systems Science and Cybernetics, IEEE Transactions on, vol. 2, no. 1, pp. 22-26, 1966.

[7] C. Bisdikian, L. Kaplan, M. Srivastava, D. Thornley, D. Verma, and R. Young, "Building principles for a quality of information specification for sensor information," in IEEE Intl. Conf. on Information Fusion (FUSION), July 2009, pp. 1370-1377.

[8] D. Turgut and L. Bölöni, "A pragmatic value-of-information approach for intruder tracking sensor networks," in Proc. of IEEE Int. Conf. on Communications (ICC-2012), June 2012, pp. 4931-4936.

[9] — , "IVE: improving the value of information in energy-constrained intruder tracking sensor networks," in Proc. of IEEE Int. Conf. on Comunications (ICC-2013), 2013, pp. 4953-4957.

[10] M. Dunbabin, P. Corke, I. Vasilescu, and D. Rus, "Data muling over underwater wireless sensor networks using an autonomous underwater vehicle," in Proceedings of the 2006 IEEE International Conference on Robotics and Automation, ICRA 2006, Orlando, FL, May 15-19 2006, pp. 2091-2098. 